\section{Watching cancer glow away}

\author{
By Michael J. Haas, Senior Writer
}

In recent years, noninvasive techniques for imaging the response of tumors to therapy have moved upstream from diagnostic to drug development settings. However, commonly used techniques like MRI provide only structural information, such as tumor volume, which is not always an accurate readout of a compound's efficacy. Functional imaging techniques like fluorodeoxyglucose PET provide a more accurate readout of tumor response by tracking biochemical processes on or within tumor cells. But functional imaging agents are expensive to produce, which limits their use.

Researchers at Vanderbilt University and Vanderbilt-Ingram Cancer Center have identified a functional imaging agent that is easier to produce than existing agents and just as effective at monitoring the response of tumors to therapy, as reported in Nature Medicine. The research team described a seven-residue peptide biomarker that differentiated between tumors that did and did not respond to treatment with a VEGF tyrosine kinase inhibitor (TKI). ${ }^{1}$

"We have not found a single VEGF TKIresponding cancer type that did not bind this peptide," said team leader Dennis Hallahan, professor of cancer research and chairman of radiation oncology at the university. "We studied cancer originating in the brain, lung, colon, skin, breast and prostate. The peptide was able to detect a response in each of these cancers," he told SciBX.

Using a phage display library owned by The Scripps Research Institute, Hallahan's team identified the peptide by screening for candidates that bound the vasculature of tumors responsive to VEGF tyrosine kinase inhibition. The peptide is known only by its sequence, HVGGSSV.

In mice with tumors, fluorescence images showed that fluorophore reporter-tagged HVGGSSV specifically bound to tumors responding to treatment with radiation and Sutent sunitinib, an inhibitor of multiple receptor tyrosine kinases. Sutent is marketed by Pfizer Inc. for renal cell carcinoma (RCC) and gastrointestinal stromal tumors.

The images were taken 24 hours after each injection of HVGGSSV.

Hallahan's team obtained similar results using three other VEGF TKIs: semaxanib, also from Pfizer, and PTK787 and AEE788, both from Novartis AG. Taken together, the results for all four compounds suggested that peptide binding was specific to the action of VEGF TKIs, not one particular compound.
In 2002 Pfizer terminated development of semaxanib, a small-molecule inhibitor of the VEGF receptor kinase insertion domain receptor, after it failed in a Phase II trial in advanced colorectal cancer. ${ }^{2}$

According to clinicaltrials.gov, Novartis has PTK787 in a Phase I trial in wet age-related macular degeneration and has AEE788 in two trials: a Phase I study in advanced cancer and a Phase I/II study in combination with the company's Certican everolimus mammalian target of rapamycin inhibitor (mTOR inhibitor) in recurrent or relapsed glioblastoma multiforme.

Histological analyses by the Vanderbilt team showed the peptide bound to the microvascular endothelia of tumors, leading the researchers to hypothesize in their paper that HVGGSSV binds to a protein "that is unveiled during endothelial response to therapy" with TKIs.

"We are currently proving the hypothesis that this peptide binds to an endothelial surface receptor," Hallahan told SciBX. He declined to discuss the results of that experiment.

In the paper, Hallahan's team said HVGGSSV is more easily synthesized than Annexin V (AnxV), a 319-amino-acid protein that binds to phosphatidylserine-which is highly expressed on the surface of cells undergoing apoptosis. AnxV has been used experimentally for the last 15 years to image how tumors respond to therapies. ${ }^{3,4}$

The Vanderbilt researchers also said HVGGSSV has faster clearance from the circulation than $\mathrm{AnxV}$, which allows for more rapid imaging of tissues and organs. Hallahan told SciBX that HVGGSSV clears quickly enough to allow imaging in about 24 hours.

However, Chris Reutelingsperger, associate professor of biochemistry at the University Maastricht's Cardiovascular Research Institute Maastricht, said the research team's statement about AnxV clearance "is rather subjective."

He pointed to a 2003 paper by Gerrit Kemerink and colleagues at University Hospital Maastricht, University Hospital Gasthuisberg, the University of Leuven and Theseus Imaging Corp., a subsidiary of North American Scientific Inc., which showed, "The clearance from the circulation occurs with a half-life of about 24 minutes." 5

Hallahan said the major obstacle to the use of $A n x V$ is its synthesis. "The problem with GMP production of large proteins like Annexin V is that they are produced by cells in culture," he said. "In contrast, small peptides like HVGGSSV can be synthesized [using] solid-state engineering," which should reduce production costs.

\section{Picturing applications}

Companies and institutions contacted by SciBX had mixed opinions on whether HVGGSSV will have greater utility in clinical or preclinical research. However, the consensus is that the specific properties of the peptide require further study.

David Chang, VP of oncology therapeutics clinical development at Amgen Inc., said the Nature Medicine paper described a "very prelimi- 
nary but a very interesting finding. There is a lot of interest in this kind of imaging, but additional details need to be worked out-for instance, the specificity of the peptide to different cancer treatments and in other cancers."

He noted that the researchers have not yet demonstrated whether the peptide targets endothelial or tumor cells. "Having an agent that targets endothelial cells - regardless of tumor type-could have broad utility. But if the peptide is shown to target tumor cells, then its utility might be limited."

Chang also expressed reservations about the use of functional imaging agents like AnxV and HVGGSSV in clinical and diagnostic settings.

"Ultimately techniques like this are interesting because it would be helpful to know whether a patient is responding to angiogenic agentswhich requires that the patient be exposed to treatment," he said. "But this approach is not as ideal as predicting whether a tumor will respond to treatment before exposing the patient to a drug."

Nevertheless, he said the findings of Hallahan's team "are the types of discoveries that Amgen would pay attention to" for potential use in its preclinical programs.

Amgen has two antiangiogenic compounds in clinical development. AMG 706, a VEGF receptor inhibitor, is in a Phase III trial for non-small cell lung cancer (NSCLC). AMG 386, a recombinant Fc peptide fusion protein targeting tyrosine kinase receptor 2 (Tie2), is in multiple Phase II trials for renal, ovarian, gastric and breast cancer tumors.

"Preclinical development of novel anticancer agents will benefit from such molecular imaging probes both by speeding up the process of development and increasing the probability of clinical success of experimental drugs," said Reutelingsperger.

He said HVGGSSV's use would likely be limited to compounds that induce the expression or exposure of the peptide's unknown target during treatment.

Binh Nguyen, CMO and VP of clinical development at Tigris Pharmaceuticals Inc., said HVGGSSV “would be a very useful technology for early preclinical research. If a company has a series of analogs of an anti-VEGF compound, it could screen that series with this technology to see which compounds are the most promising and most potent."

Nguyen added: "It would be important to know the exact function of this HVGGSSV peptide on the endothelial membrane proteins."

Tigris has GFB-204, a calixarene-derived VEGF receptor inhibitor, in preclinical development to treat solid tumors.

Clive Wood, EVP of discovery research and CSO at Dyax Corp., said it was "impressive" that a seven-residue peptide could achieve such a specific readout so quickly.

"It's unusual to use this kind of imaging in preclinical work," he said. "More effective and validated imaging endpoints could change the course of preclinical studies: experiments that now take weeks or months to give feedback could take as little as two or three days."

He would like to see the peptide tested with other classes of anticancer drugs, "just to see what happens-in Avastin, for instance," he said. "It would be very interesting to know what other drugs can work with this peptide."
Wood also said the peptide could help determine how best to introduce a drug candidate into established anticancer regimens before entering clinical trials.

"You only get a few chances to get this right," he said. "Imaging drug combinations in a preclinical setting could better predict effective combinations in a clinical setting, increasing the likelihood of success in development."

As an example, Wood cited Dyax's DX-2240, a human mAb that targets tyrosine kinase receptor 1 (Tie1) on tumor neovasculature. On its own, he said, the antibody has "modest to strong activity" in some preclinical models, but in specific combination dosing regimens, DX2240 increases the antitumor activity of other cancer therapies such as Avastin and Nexavar.

Last month, Dyax granted sanofi-aventis Group exclusive worldwide rights to develop and commercialize DX-2240. The compound is in preclinical development to treat cancer. ${ }^{6}$

Genentech Inc. markets Avastin bevacizumab, a humanized mAb against VEGF, in the U.S. for breast, non-small cell lung, colorectal and renal cancers. Roche markets the drug elsewhere.

Nexavar sorafenib, from Onyx Pharmaceuticals Inc. and Bayer AG, is an inhibitor of multiple kinases, including VEGF receptors. It is approved in the U.S. and EU for the treatment of both hepatocellular carcinoma and RCC.

Conversely, Dana Aftab, VP of translational research at Exelixis Inc., said the preclinical utility of the peptide would be limited if it can only detect tumor response to VEGF kinase inhibitors.

"I don't know of any companies that are still actively focused on the discovery and preclinical characterization of new compounds that target VEGF receptors for antiangiogenesis," Aftab said. "In general, the majority of these compounds have already moved into the clinic. So many companies, including Exelixis, are now focused on developing drugs that target enzymes or pathways that are more directly involved in the proliferation and survival machinery within tumor cells, such as MET, RAF, MEK, PI3K and others."

Aftab agreed with others that the peptide's utility may also depend on whether it binds a target on endothelial or tumor cells.

"If the peptide binds an endothelium-specific protein, then it may only be applicable to treatments that directly impact the tumor vasculature," he said. "If the peptide is recognizing a nonendothelial stromal or basement membrane component, or a tumor cell component resulting from cell death, it may be applicable to most tumors because these components are fairly well conserved."

Likewise, he said the peptide might have less utility if it bound a protein specific to only certain tumor cells. He doubted this was the case, but said it was unclear from the paper's supplemental data whether the peptide was detecting an endothelial protein or a tumor cell protein.

Aftab also was interested in seeing data obtained in a wider range of animal models. "In the paper, the peptide seemed to give the best response in the Lewis lung carcinoma mouse model, but this model is not derived from a human cancer," he said. "The researchers used a few human cancer models; these seemed to respond but not as robustly." 
Data from a larger array of human tumor xenografts and genetic mouse models, and from a wider variety of therapeutic agents, would give a better picture of the specificity of the peptide, Aftab said.

"A fuller set of data would help to determine how broadly applicable the peptide is," he noted. "And those data would need to be characterized very carefully so that researchers could determine whether the peptide would work in their preferred models."

Aftab added: "I would need this kind of information before I could think about using the peptide preclinically."

Aftab said the peptide showed the most promise for clinical applications. "It would be a real benefit to have an imaging technique that could show a treatment effect after one or two weeks-instead of having to use traditional criteria that require generally two or more months of treatment," he said.

Exelixis has several anticancer drugs in clinical trials. The company's lead compound, XL647, which targets VEGF receptor, epidermal growth factor receptor (EGF receptor) and EGF receptor 2 (HER2), will enter Phase III trials for NSCLC this year.

XL184, which targets MET, RET and VEGF receptor, is in Phase I for medullary thyroid cancer and Phase I/II for lung cancer. The company will take XL184 into a pivotal study in medullary thyroid cancer later this year and into a Phase II trial in glioblastoma in $2 \mathrm{H} 08$.

Hallahan said the team plans to take HVGGSSV into clinical trials but has not set a date to begin a Phase I study.

In the meantime, the team is experimenting with three radioisotopes of iodine, any of which could replace the fluorophore tag as a reporter on HVGGSSV. These radioiodine reporters are conjugated to the peptide by three tyrosine residues, an addition that Hallahan said does not affect the pharmacokinetic profile of the peptide.
He said the team is interested in using ${ }^{131}$ I for the clinical trials because it would deliver radiation to the tumor while imaging it.

Hallahan said that Vanderbilt University has applied for a provisional patent on the findings reported in Nature Medicine and is negotiating the licensing of development rights with Cumberland Pharmaceuticals Inc.

\section{REFERENCES}

1. Han, Z., et al. Nat. Med.; published online Feb. 24, 2008; doi:10.1038/ $\mathrm{nm} 1691$

Contact: Dennis E. Hallahan, Vanderbilt University, Nashville, Tenn. e-mail: dennis.hallahan@vanderbilt.edu

2. Maggos, C. BioCentury 13(8) A8; Feb. 142005

3. Boersma, H. et al. J. Nucl. Med. 46, 2035-2050 (2005)

4. Corsten, M. et al. Cancer Res. 66, 1255-1260 (2006)

5. Kemerink, G. et al. J. Nucl. Med. 44, 947-952 (2003)

6. Ward, M. BioCentury 16(8) A11; Feb. 182008

COMPANIES AND INSTITUTIONS MENTIONED

Amgen Inc. (NASDAQ:AMGN), Thousand Oaks, Calif. Bayer AG (FSE:BAY), Leverkusen, Germany Cumberland Pharmaceuticals Inc., Nashville, Tenn. Dyax Corp. (NASDAQ:DYAX), Cambridge, Mass. Exelixis Inc. (NASDAQ:EXEL), South San Francisco, Calif. Genentech Inc. (NYSE:DNA), South San Francisco, Calif. North American Scientific Inc. (NASDAQ:NASI), Chatsworth, Calif. Novartis AG (NYSE:NVS; SWX:NOVN), Basel, Switzerland Onyx Pharmaceuticals Inc. (NASDAQ:ONXX), Emeryville, Calif. Pfizer Inc. (NYSE:PFE), New York, N.Y.

Roche (SWX:ROG), Basel, Switzerland sanofi-aventis Group (Euronext:SAN; NYSE:SNY), Paris, France

The Scripps Research Institute, La Jolla, Calif.

Tigris Pharmaceuticals Inc., Bonita Springs, Fla. University Hospital Gasthuisberg, Leuven, Belgium University Hospital Maastricht, Maastricht, The Netherlands University of Leuven, Leuven, Belgium

University Maastricht, Maastricht, The Netherlands Vanderbilt-Ingram Cancer Center, Nashville, Tenn. Vanderbilt University, Nashville, Tenn. 